\title{
A New Variant of Combined Pulmonary Fibrosis and Emphysema from Long-Term High-Dose of Glucocorticoid Therapy: A Case Report
}

\author{
Qigang Zeng, Chenxia Duan, Yong Dai \\ Guangdong Hospital of Integrated Traditional Chinese and Western Medicine, Foshan, China \\ Email: zqg20037@163.com
}

How to cite this paper: Zeng, Q.G., Duan, C.X. and Dai, Y. (2017) A New Variant of Combined Pulmonary Fibrosis and Emphysema from Long-Term High-Dose of Glucocorticoid Therapy: A Case Report. Case Reports in Clinical Medicine, 6, 301307.

https://doi.org/10.4236/crcm.2017.612035

Received: June 28, 2017

Accepted: December 2, 2017

Published: December 5, 2017

Copyright $\odot 2017$ by authors and Scientific Research Publishing Inc. This work is licensed under the Creative Commons Attribution International License (CC BY 4.0).

http://creativecommons.org/licenses/by/4.0/

\begin{abstract}
Recent studies have described the combination of both pulmonary emphysema and idiopathic interstitial lung disease (ILDs) by means of high-resolution computed axial tomography (HRCT). Definition of this syndrome was first named by Cottin as combined pulmonary fibrosis and emphysema (CPFE). Functional and radiological findings have showed that these patients are suffering from severe breathlessness, but whose pulmonary functional tests revealed no signs of obstruction, normal static lung volumes, and depressed DLco, most with a history of smoking [1] [2]. The radiological and endoscopic studies especially show that these patients have both areas of upper-lobe predominant emphysema and lesions compatible with fibrosis in both lung bases [3]. No prior research has reported any cases of such condition in person with no prior history of smoking as well as long-term high-dose of glucocorticoid therapy. In this case report, we discuss the presentation, diagnosis, and management of a 53-year-old non-smoker with increasing shortness of breath with a long-term high-dose of glucocorticoid therapy discovered to have an abnormal variant or presentation of CPFE. The cause of disease was attributed to a certain history of smoking in most studies; other potential risk factors have yet to be properly analyzed. This clinical report features a special case about the problem and solution surrounding this issue.
\end{abstract}

\section{Keywords}

Emphysema, Pulmonary Fibrosis, High-Dose of Glucocorticoid Therapy, Lung Diseases

\section{Introduction}

Pulmonary emphysema and idiopathic pulmonary fibrosis are once to be recog- 
nized as separate disease. Since the advent of HRCT, the combination of these two statuses has been proven to be a prevalent and distinct entity rather than a rare coincidence. Since it's first described in 1990s, patients with fibrosis and upper lobe emphysema show some special characteristics such as severe breathlessness, low DLco but with preserved lung volumes [4]. Studies have shown that patients with CPFE are mostly presented with tobacco exposure. They are predominantly male, with a history of heavy tobacco exposure, and usually present with severe breathlessness and cough. Physical examination reveals "Velcro" crackles at the lung bases and digital clubbing [3]. However, in this case, the patients were discovered with non-smoking history, but a long-term high-dose of glucocorticoid therapy in treating gouty arthritis. This brings into a new question, whether there is an undiscovered risk factor or a new condition, which presents differently and may be the result of another risk factor, other than smoking.

\section{Case Summaries}

A 53-year-old male with non-smoking history but a long-term medical history of gouty arthritis presented to our department. The main symptoms of the patient were long history of dyspnea exacerbation (from stage I to stage III), associated with productive cough consisting of yellow phlegm and persistent chest pain. The clinical manifestations gradually increased a week prior for which he visited the primary medical doctor and was treated for pneumonia. His symptoms continued to persist and gradually worsen at which point he was also treated with a long-term (lasting for 1.5 years) high-dose of glucocorticoid therapy (80 mg of methylprednisolone per day) for gouty arthritis. His other symptoms however continued to progress and consisted of fever, cough, shortness of breath, chest pain and severe attacks in both joints. The patient is a non-smoker, drinks alcohol socially, and works in a paper mill.

On physical examination, the patient was tachycardia with a heart rate of 114 , blood pressure of $137 / 74 \mathrm{mmHg}$, respiratory rate of $30 / \mathrm{min}$, and was saturating $86 \%$ on room air. Following above symptoms were some nonspecific illness such as mild fever, tender lymph nodes, sore throat, headaches, weakness, muscle and joint pain. He was sent to the ED in Monday, and by Wednesday his fever was $101.3^{\circ} \mathrm{F}$ and the respiratory distress and chest pain was unbearable. Chest auscultation revealed scattered to diffuse crackles and rhonchi. Laboratory data demonstrated white blood cell of 22.8 (normal range from $4-10 \times 10^{9} / \mathrm{L}$ ), absolute neutrophil count of 19.9 (normal range from $1.2-6.79 \times 10^{9} / \mathrm{L}$ ), neutrophil ratio of 87.1 (normal range from 50\% - 70\%), procalcitonin of $19.12 \mathrm{ug} / \mathrm{L}$ (normal range from $0-0.5 \mathrm{ug} / \mathrm{L}$ ) and C-reactive protein level was over $200 \mathrm{mg} / \mathrm{L}$ (normal range $\leq 10 \mathrm{mg} / \mathrm{L}$ ). $\mathrm{PH}$ was $7.5, \mathrm{Po}_{2}$ was $9.1 \mathrm{kPa}$ (normal range from 10.66 - $13.33 \mathrm{kPa}$ ), $\mathrm{Pco}_{2}$ was $4.8 \mathrm{kPa}$ (normal range from $0.47-6.00 \mathrm{kPa}$ ). Meanwhile, the vital signs, blood glucose and lactate, blood electrolytes and troponins were negative. D-dimer was elevated at $1.23 \mathrm{mg} / \mathrm{L}$ (normal range $<0.5$ 
$\mathrm{mg} / \mathrm{L}$ ) and HRCT was conducted to rule out pulmonary embolism. However, there were shadows and fibrosis cord changes through HRCT, the bullae formation in the upper lung fields, with local fibrosis were observed around the basal segments of the lung fields, suspicious for an infectious or inflammatory process (Figure 1 and Figure 2).

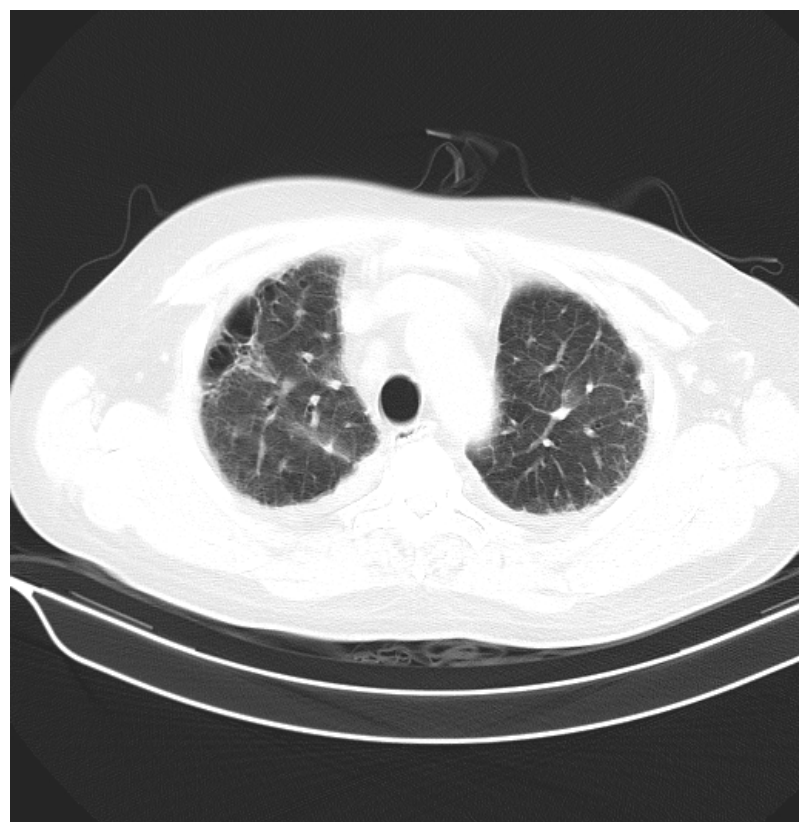

Figure 1. Chest HRCT showed centrilobular emphysema and bullae in the upper lobes.

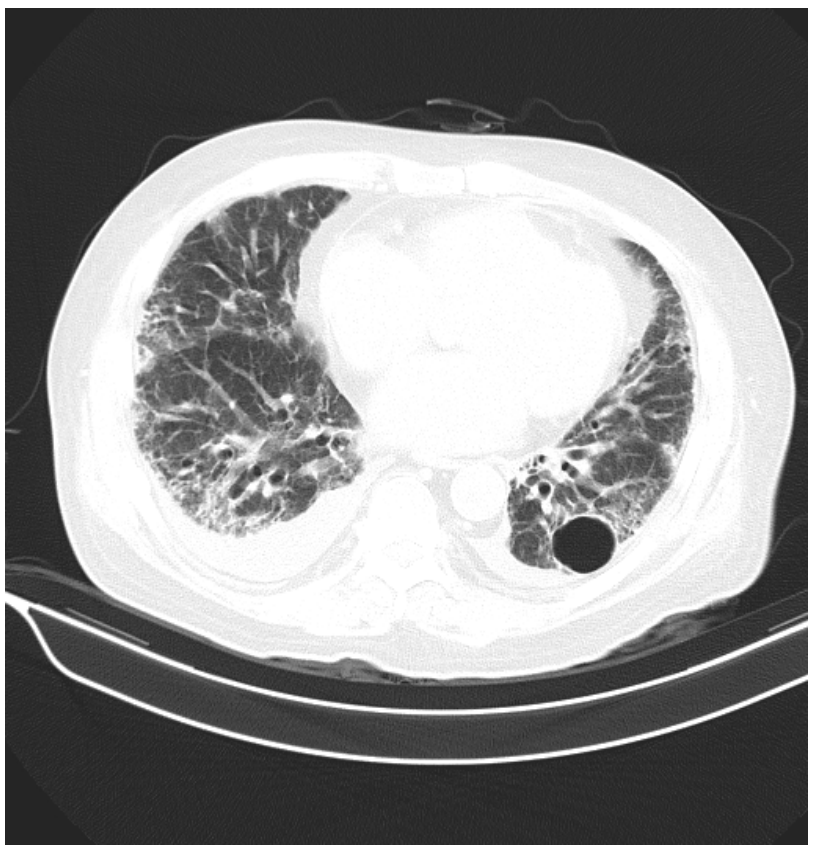

Figure 2. Chest HRCT showed honey combing, scissural distortion and ground glass opacities in the bases and the periphery of the lungs. 
He had undergone almost a week of inpatient therapy for the management of severe community acquired pneumonia with piperacillin-tazobactam combined with levofloxacin for 5 days. Patient was found to have positive result of methicillin-resistant staphylococcus aureus (MRSA) sputum culture and medications were adjusted according to sensitive antibiotics therapy. He was treated for severe bacterial infection of lower respiratory tract with Meropenem combined with Vancomycin through intravenous infusion. Inflammation markers showed obviously decrease in the first week, but patient continued to have persistent hypoxia and continued to desaturate to $85 \%$ upon ambulation. Bronchoscopy was also received but no significant mass or lesion was found to biopsy and his postoperative diagnosis remained as non-resolving pneumonia. The application of noninvasive positive airway pressure ventilation combining with methylprednisolone and antibiotics was proved to be effective on this patient, he was still suffering from diffuse bilateral crackles and productive cough. Patient was found to have leukocytosis as high as 21.0, but this could be attributed to the steroid treatment. The patient continued to deteriorate and other autoimmune antibodies including antineutrophil cytoplasmic autoantibody (cANCA), perinuclear anti-neutrophil cytoplasmic antibodies (pANCA), antinuclear antibody (ANA) were negative.

After completing the course of antibiotics, he was continue to receiving glucocorticoid sequential treatment and lung findings remained unchanged. Besides, the Borg scores, ventilation function were measured both in exacerbation stage. Pulmonary function tests before acute exacerbation were significant for a forced vital capacity (FVC) moderately reduced at $1.58 \mathrm{~L}$ (63\%), forced expiratory volume in 1 second (FEV1) severely reduced at $1.09 \mathrm{~L}$ (59\%), FEV1/FVC ratio was normal at 59\%. Flow volume loop demonstrated a combined obstructive and restrictive contour. Lung volumes showed total lung capacity (TLC) reduced at $1.86 \mathrm{~L}(52.9 \%)$, and residual volume (RV) reduced at $0.41 \mathrm{~L}(41 \%)$. The diffusion capacity (DLco) was also severely reduced at $34 \%$. All index showed no significant changes after the inhalation. These findings were suggestive of combined severe, irreversible obstructive and restrictive lung disease with severely reduced diffusion capacity. Repeat CT scan was performed after the anti-inflammatory treatment, which showed minimal improvement and continue to suggest pneumonia and possibly underlying interstitial lung disease. The patient was suggested for biopsy of the lung tissues, but an allergy history of anesthetics was absolute contraindications for this patient.

He maintained the medical therapy with Methylprednisolone $20 \mathrm{mg} / \mathrm{day}$ and Acetylcysteine $1800 \mathrm{mg} / \mathrm{d}$, the patient clinically improved over another two weeks. His pulse oximetry on room air improved around 90\% and patient had spontaneously breathing on home oxygen therapy without respirator. An ongoing follow-up study is 2 years in which the patient had a stable dyspnea (stage II of the NYHA) and slight hypoxemia with oxygen at home for less than 20 hours per day. 


\section{Discussion}

Whether CPFE represents a unique disease entity or a coincidence of two pulmonary diseases related to cigarette smoking is as yet unclear. So far, the diagnosis of the CPFE syndrome relies on HRCT imaging and pathology [5]. The descriptions of CPFE include some certain features as male sex, a history of cigarette smoking, relatively preserved spirometric values, and decreased diffusing capacity of lung for carbon monoxide (DLco) [6].

The pathogenesis of CPFE has not been fully described and it's still unclear whether two distinct pathological change progress independently or if one results from the other [7]. HRCT scanning is an essential part for diagnosing CPFE. Anyway, the relative contributions of pathological characteristics vary among patients with CPFE [8]. Diagnosis of CPFE on HRCT is still the certain method to assess the degree of emphysema and fibrosis, which may make the progress effective and accurate [9]. In this case presentation unlike typical CPFE, emphysema was found in the lower lobe on this patient, CT scan was unremarkable for emphysematous changes, lung volume changes were present, and the patient had a long-term high-dose of glucocorticoid therapy instead of smoking history. It brings into question, whether long-term high-dose of glucocorticoid therapy or some form of steroid medicaitons may result in a severe variant or subtype of CPFE, which can present suddenly and has yet to be discovered.

What make this case report even more unusual are the findings found on high contrast CT imaging. As previously mentioned the normal defining factor of $\mathrm{CPFE}$ consists of upper lobe emphysema and lower lobe fibrosis with reduced carbon monoxide transfer (DLco) [10]. However, on imaging of our patient there was emphysema both in upper and lower lobe. Some findings consistent with pulmonary fibrosis located in the lower lobes of the lungs. It is particularly unusual that emphysematous changes and fibrotic lesions located in the same lower region as it has not been reported before.

Pulmonary hypertension $(\mathrm{PH})$ is a common complication during the course of disease [11]. CPFE accompanied with pulmonary hypertension could have a much higher impact on mortality, prognosis and economic evaluation [12]. Cardiac ultrasonography was done in this case and it showed dilated right chambers and pulmonary hypertension with systolic pulmonary arterial pressure of $84 \mathrm{~mm}$ Hg. Unfortunately, patient refused to accept any repeat testing and future treatment evaluation because of economical restriction. Furthermore, pulmonary function testings including FEV1, TLC, and FVC are usually within normal ranges or slightly abnormal in typical CPFE changes [7], this case had showed a significant and sustained decline in above parameters of lung function, as the interaction of emphysema and fibrosis changes constraints finally would have attained an imbalance status in the end-stage of CPFE. However, the two pathologies still will result in a negative synergic effect on gas exchange, which results in a significant decline in DLco. Again, these findings other than the reduced DLco contraindicate what was seen in our patient. Whether the parame- 
ters of lung function would still kept at the normal level or near normal level in the end-stage or a completely complex data type would possibly be discovered remains questionable.

There is no effective treatment of this disorder. The best option is smoking cessation, home oxygen therapy and lung transplantation. Bronchodilators are frequently prescribed to patients with a positive response in their pulmonary function test. Home oxygen therapy can also be implemented and may be necessary. However, oral corticosteroids and immunosuppressant have been considered an option in the acute episode of CPFE. In this case, the patient was a non-smoker and found to be at an end-stage, he was later recommended to a transplant center. As our patient has an allergy history of anesthetics and found to be at an end-stage, he refused to accept the unilateral lung transplantation surgery. Meeting the criteria, continuous administration of middle flow home oxygenation was recommended to patient for at least 15 hours per day. Although the application of high dose methylprednisolone during acute attack and sequential treatment in stable phase was proved to be effective, the benefit of patients still need to be further confirmed. Moreover, immunosuppressant have been considered an option in the setting of CPFE, the application of pirfinedone (PF) therapy were used for only a short time, while side effects including mild headache, shivers, nausea and vomiting happened occasionally. Additionally, PF therapy cost much, putting a heavy burden on the patient himself, which decreased the application and accessibility of these drugs heavily.

The growing number of published papers about CPFE demonstrates the increasing interest in this newly defined disease, which encompasses a particular clinical, functional, and radiological profile. Special attention for physicians when meeting patients treated with a long course of antibiotics or steroids with minimal improvement in their condition, it should be taken into consideration. In this case presentation unlike typical CPFE, emphysema was found both in the upper and lower lobe upon HRCT scan, pulmonary function testing had showed a significant and sustained decline, and the patient had a certain history of long-term high-dose of glucocorticoid therapy instead of smoking. It brings into question, whether long-term high-dose of glucocorticoid therapy or glucocorticoid therapy itself may result in a severe variant or subtype of CPFE, which can present suddenly and has yet to be discovered.

In order to respect the patient's privacy, the clinical photos or details of the case, and the consent articles were obtained after signing the informed consent by the patients and his close relatives.

\section{Conflict of Interest}

The authors declare that there is no conflict of interest regarding the publication of this paper.

\section{References}

[1] Cottin, V., Nunes, H., Brillet, P.Y., Delaval, P., Devouassoux, G., Tillie-Leblond, I., 
et al. (2005) Combined Pulmonary Fibrosis and Emphysema: A Distinct under Recognised Entity. The European Respiratory Journal, 26, 586-593. https://doi.org/10.1183/09031936.05.00021005

[2] Kitaguchi, Y., Fujimoto, K., Hanaoka, M., Kawakami, S., Honda, T. and Kubo, K. (2010) Clinical Characteristics of Combined Pulmonary Fibrosis and Emphysema. Respirology, 15, 265-271. https://doi.org/10.1111/j.1440-1843.2009.01676.x

[3] Sarkavas, J.A., Karkhanis, V.S. and Joshi, J.M. (2013) Pulmonary Fibrosis and Emphysema. Indian Journal of Chest Diseases and Allied Sciences, 55, 97-99.

[4] Auerbach, O., Garfinkel, L. and Hammond, E.C. (1974) Relation of Smoking and Age to Findings in Lung Parenchyma: A Microscopic Study. Chest, 65, 29-35. https://doi.org/10.1378/chest.65.1.29

[5] Zendah, I., Gharsalli, H., Khattab, A. and Ghedira, H. (2011) The Syndrome of Combined Pulmonary Fibrosis and Emphysema. Tanaffos, 10, 59-62.

[6] Mejía, M., Carrillo, G., Rojas-Serrano, J., Estrada, A., Suárez, T., Alonso, D., et al. (2009) Idiopathic Pulmonary Fibrosis and Emphysema: Decreased Survival Associated with Severe Pulmonary Arterial Hypertension. Chest, 136, 10-15. https://doi.org/10.1378/chest.08-2306

[7] Lin, H.J. and Jiang, S.P. (2015) Combined Pulmonary Fibrosis and Emphysema (CPFE): An Entity Different from Emphysema or Pulmonary Fibrosis Alone. Journal of Thoracic Disease, 7, 767-779.

[8] Dias, O.M., Baldi, B.G., Costa, A.N., et al. (2014) Combined Pulmonary Fibrosis and Emphysema: An Increasingly Recognized Condition. Jornal Brasileiro De Pneumologia, 40, 304-312. https://doi.org/10.1590/S1806-37132014000300014

[9] Kitaguchi, Y., Fujimoto, K., Hanaoka, M., et al. (2010) Clinical Characteristics of Combined Pulmonary Fibrosis and Emphysema. Respirology, 15, 265-271. https://doi.org/10.1111/j.1440-1843.2009.01676.x

[10] Wiggins, J., Strickland, B. and Turner-Warwick, M. (1990) Combined Cryptogenic Fibrosing Alveolitis and Emphysema: The Value of High Resolution Computed Tomography in Assessment. Respiratory Medicine, 84, 365-369.

[11] Cottin, V. and Cordier, J.-F. (2012) Combined Pulmonary Fibrosis and Emphysema in Connective Tissue Disease. Current Opinion in Pulmonary Medicine, 18, 418-427. https://doi.org/10.1097/MCP.0b013e328356803b

[12] Seeger, W., Adir, Y., Barberà, J.A., et al. (2013) Pulmonary Hypertension in Chronic Lung Diseases. Journal of the American College of Cardiology, 62, D109-D116. https://doi.org/10.1016/j.jacc.2013.10.036 